\title{
Aspectos Inmunohistoquímicos y Alteraciones Moleculares en Quistes y Tumores Odontogénicos
}

Aspects of immunohistochemical and molecular alterations in odontogenic cysts and tumors

Fecha de Recepción 25 de abril de 2012
O aspectos imuno-histoquímica e molecular alteraçōes em cistos e tumores odontogênicos
Profesor Titular Cátedra Anatomía Patológica. E-mail: arvallejos@gmail.com

M. S. Briend Profesora Adjunta Cátedra Anatomía Patológica.

M. S. Almirón Jefe Trabajos Prácticos Cátedra Anatomía Patológica.

V. R. Fernández Profesor Adjunto Cátedra Estomatología Clínica.

P. L. Fortín Adscripto para Perfeccionamiento en la Disciplina y Iniciación en la Investigación

J. M. Vallejos

Adscripto para Perfeccionamiento en la Disciplina.

\section{Lugar de Trabajo}

Cátedra de Anatomía Patológica.

Facultad de Odontología. UNNE

Av. Libertad $5450-2^{\circ}$ piso - Int 130

Corrientes (Capital)
Aceptado para su publicación 30 de agosto de 2012

\section{Resumen}

Los quistes y tumores odontógenos representan una importante causa de asistencia a la consulta. Si bien en la mayoría de las mismas no se conoce su etiopatogenia, hemos revisado sus antecedentes y la valoramos con la información actual.

De las técnicas moleculares, la inmunohistoquímica permite observar la presencia de oncoproteínas, y/o alteraciones en los receptores de la superficie celular o nuclear, para detectar las alteraciones genéticas y moleculares que se vayan sucediendo y que, libradas al azar, producen la aparición de una población clonal de una neoplasia.

Es nuestro interés revisar las inmunotinciones de utilidad en quiste $y$ tumores odontogénicos y relacionarlas con el estado actual del conocimiento sobre las alteraciones moleculares que subyacen en cada una de ellas y rever el estado actual de las mismas.

\section{Palabras Claves}

Quistes - tumores odontogénicos - inmunotinción - alteraciones moleculares.

\section{Summary}

Cysts and odontogenic tumors represent an important cause of attendance at the consult. While most of the same etiology is unknown, we have reviewed its history and value with current information.

Among the molecular techniques, immunohis- 
tochemistry allows to observe the presence of oncoproteins, and/or alterations in cell or nuclear surface receptors, in order to detect genetic and molecular changes that are happening and that, left to chance, may produce the appearance of a clonal population of a neoplasia.

Our interest is to review the utility immunostaining in cyst and odontogenic tumors and relate to the current state of knowledge about the molecular alterations that underlie each and review the current state of them.

\section{Keywords}

Cysts - odontogenic tumors - immunostaining molecular alterations.

\section{Resumo}

Cistos e tumores odontogênicos representam uma importante causa de seu comparecimento à consulta. Enquanto a maioria da mesma etiologia é desconhecida, nós analisamos a sua história e valor com informações atuais.

De técnicas moleculares, imuno-histoquímica pode-se observar a presença de oncoproteínas, e/ou alterações nos receptores de superfície celular ou nuclear, para detectar alterações genéticas e moleculares que estão acontecendo e que, deixado ao acaso, produzir a aparência de uma população clonal de uma neoplasia.

Nosso interesse é revisar a imunomarcação utilidade em cistos e tumores odontogênicos e se relacionam com o estado atual do conhecimento sobre as alterações moleculares que fundamentam cada um e analisar o estado actual da mesma

\section{Palavras chaves}

Cistos - tumores odontogênicos - imunorreatividade - alterações moleculares.

\section{Introducción}

Los quistes representan una de las principales causas de la pérdida de la integridad del componente maxilo-facial, ocasionando trastornos funcionales $y$ estéticos de variable intensidad e inclusive la formación de neoplasias a partir de los mismos.

Los tumores odontógenos son poco frecuentes y algunos extremadamente raros, representan aproximadamente el $2-3 \%$ de las neoplasias en general. Dentro de las patologías tumorales que comprometen a los maxilares alcanzan al el $85 \%$ y el resto corresponden a los del tejido óseo. Incluyen hamartomas, neoplasias benignas, algunas agresivas y muy escasas las malignas.

Las localizaciones exclusivas en los maxilares deben ser tenidas en cuenta en los diagnósticos diferenciales con lesiones ubicadas en este sitio. En cuanto a la etiopatogenia, está claro el origen de los quistes a partir de restos epiteliales y de algunos tumores a partir de los mismos; pero la mayoría todavía se desconoce.

Para comprender el valor de las técnicas inmunohistoquímicas y las alteraciones moleculares responsables del desarrollo de los tumores, es necesario, ubicarse inicialmente, en el contexto del ciclo celular, pues su desregulación y los acontecimientos moleculares que acontecen durante la carcinogénesis son determinantes de los procesos patológicos por ellos generados.

Se demostró la transformación desde los epitelios de quistes odontogénicos como los casos de ameloblastomas uniquísticos y/o los carcinomas escamosos intraóseos a partir de restos de células epiteliales aprisionadas en los maxilares durante el proceso de embriogénesis y que están sujetos a la acción de agentes carcinogenéticos usuales, capaces de desarrollar estas neoplasias. En estos últimos años han surgido nuevos conocimientos sobre la etiopatogenia, a partir de sucesos moleculares implicados en la proliferación celular que se han descubierto a través de los denominados protooncogenes. La presencia de defectos en estos genes reguladores de la proliferación celular, los convierten en oncogenes que no cumplen las funciones reguladoras específicas y son responsables del surgimiento de clones de células neoplásicas. Las mismas se producen por alteraciones o fallas en cualquier punto de las vías de transducción de señales y consecuentemente se genera inestabilidad genética, por defectos en los puntos de control y la presencia de oncogenes, responsables de la aparición de las proliferaciones neoplásicas.

Es nuestro interés revisar las inmunotinciones actualmente utilizadas para poner de manifiesto y relacionar con en el estado actual del conocimiento las alteraciones genéticas y moleculares responsables de los mismos. 


\section{Desarrollo}

Actualmente a través de la oncología molecular se han logrado identificar más de $\mathbf{2 0 0}$ genes implicados en la génesis de estas neoplasias mediante estudios experimentales $y$ en grupos familiares. Nuestra comprensión de la alteraciones moleculares y genéticas asociadas con el desarrollo y la progresión de los tumores odontogénicos, incluyen entre otros a los oncogenes, genes supresores de tumores, los oncovirus, factores de crecimiento, la telomerasa, los reguladores del ciclo celular, factores reguladores de la apoptosis, reguladores del desarrollo del diente, tejidos duros relacionados con las proteínas, moléculas de adhesión celular, matriz de degradación por proteinasas, factores angiogénicos, $y$ citoquinas osteolíticas entre otras'.

Hanahan y Weinberg ${ }^{2}$, revelan que todas las neoplasias desarrolladas presentan un sello o funciones biológicas adquiridas, que expresan la complejidad de las mismas. Enuncian características que incluyen, el mantenimiento de la señalización de proliferación, la evasión del control de los genes supresores tumorales, la resistencia a la muerte celular, lo cual favorece la replicación, la inducción de la angiogénesis ${ }^{3,4,5,6}$ y la activación de la invasión y metástasis. Estas características de identidad son producidas por la inestabilidad del genoma, expresado anteriormente y en los últimos años se han agregado dos características más, la reprogramación del metabolismo energético y la evasión del control del sistema inmunológico ${ }^{7,8}$. Además es importante reconocer el papel del microambiente tumoral que contribuye ostensiblemente a la adquisición y mantenimiento del sello característico de la neoplasia y que surge de la interelación de las células neoplásicas, de las células normales y de las células del estroma tumoral. Además existen otros factores relacionados, que interactúan como: el estado general del huésped, el medio local donde se encuentra, la presencia de mediadores químicos del componente inflamatorio', la transición epitelio-mesénquima ${ }^{10}$, el rol de las células madres descriptos en los mecanismos de invasión y metástasis ${ }^{11,12}$, pues sostienen con diferentes grados de participación ese microambiente propicio para las neoplasias ${ }^{13,14,15}$.

La etiología y patogenia de los tumores odontogénicos todavía no son bien conocidas, $y$ cons- tituyen todo un desafio. Recientes estudios de Kumamoto ${ }^{16}$ han identificado alteraciones moleculares responsables del desarrollo y progresión de los mismos, que se resumen en la tabla (ver Tabla I).

Se han descriptos varios factores de crecimiento polipeptídicos que estimulan la proliferación de células normales y muchos intervienen en la tumorogénesis. Por ejemplo, como se expresó anteriormente, la mutación de los genes que codifican los factores de crecimiento puede convertirlos en oncogénicos, los cuales codifican proteínas denominadas oncoproteínas. Esto provocaría una expresión excesiva de factores de crecimiento, una proliferación descontrolada de las células o la activación persistente constitutiva sin necesidad de unirse al factor de crecimiento correspondiente. De esta manera el receptor mutante liberaría hacia la célula continuas señales que estimulan la mitosis. Ejemplo de tumores producidos por oncoproteínas por mutación genómica constituye el Tumor Odontógeno Quístico Queratinizante.

En la nueva clasificación que fue aprobada por el consenso de la conferencia realizada en Lyon, Francia (OMS/IARC) en julio del 2003 en conjunto con la preparación del volumen del Blue Book de Patología y Genética de Tumores de Cabeza y Cuello editado en 2005 por la Organización Mundial de la Salud (OMS) I7, se ha incluido al Queratoquiste Odontógeno Paraqueratinizado, dentro del grupo de los Tumores Odontogénicos, como Tumor Odontogénico Quístico Queratinizante, quedando el Queratoquiste Odontogénico Ortoqueratinizado como otra entidad separada dentro de los Quistes Odontógenos existentes. Esta última reubicación dentro de las clasificaciones en vigencia, constituye un punto de inflexión - bisagra esclarecido a través de la biología celular y molecular. Pues se ha identificado $y$ atribuido al gen PTCHI (9q22.3-3I) I8, I9,20 como principal responsable del Tumor Odontogénico Quístico Queratinizante (ex Queratoquiste) presente en el Síndrome de Carcinoma Nevoide de Células Basales (o Sindr. Gorlin-Goltz) y también otros genes como el PTCH2 y SUFU podrían estar involucrados2I,22.El PTCH2 codifica una proteína de transmembrana involucrada en la vía de señalización celular SHH/PTCH, homólogo 
Tabla I: Resumen de Moléculas Involucradas en los Tumores Odontogénicos.

\begin{tabular}{|l|l|}
\hline \multicolumn{2}{|c|}{ Molécullas Asociadas con la diferenciación de la células tumorales } \\
\hline Oncogenes & Ras, Myc \\
\hline Genes supresores tumorales & P53 \\
\hline Oncovirus & HPV, EBV \\
\hline Factores de Crecimiento & TGF-a, -b, FGF-I, -2, HGF \\
\hline Reguladores del Ciclo Celular & Ciclina DI, pl6, p 2I \\
\hline Factores relacionados con la Apoptosis & Bcl-2, Fas, p53 \\
\hline Reguladores del desarrollo del diente & SHH pathway, Wnt pathway \\
\hline Proteínas reguladoras de los tejidos duros & $\begin{array}{l}\text { Amelogenina, enamelina, osteocalcina, ena- } \\
\text { melisina, proteína morfogénica del hueso, } \\
\text { osteonectina, osteocalcina y osteopontina. }\end{array}$ \\
\hline Factores angiogénicos & IL-I, -6, TNF-a, PTHrP \\
\hline Moléculas de adhesión celular & E-selectina, ICAM-I, VCAM-, caderinas, CD44 \\
\hline \multicolumn{1}{|c|}{ Moléculas asociadas a la progresión del tumor } \\
\hline
\end{tabular}

con la proteína PTCHI. Fan y $\mathrm{col}^{23}$, identificaron mutaciones en el gen PTCH2 en una familia china con síndrome de Gorlin-Goltz, demostrando la pérdida de la función inhibitoria de este gen, en la vía de señalización celular $\mathrm{Hh}$. Por otro lado el gen SUFU es un gen supresor tumoral que predispone a los individuos a meduloblastomas, componente del síndrome. Estudios de Pastorino y colaboradores ${ }^{24}$ también identificaron una mutación de este gen, en familias PTCH-I negativas con signos y síntomas del Síndrome de GorlinGoltz. Por lo tanto, las mutaciones de los genes PTCH-2 y SUFU son detectables en un pequeño número de familias con Síndrome de GorlinGolt, en menor frecuencia que las mutaciones del gen PTCH-I.

Otros grupos de investigaciones ${ }^{25}$ han demostrado la pérdida de heterogenicidad en los genes supresores tumorales asociados a neoplasias humanas como el p 16, p53, MCC, TSLCI, LTAS2. Estos hallazgos son útiles para explicar el comportamiento agresivo del Tumor Odontogénico Quístico Queratinizante y dar mayor apoyo a su naturaleza neoplásica.

Otro ejemplo de activación, es la mutación del oncogen Ras, que a través de estudios en mamíferos se han identificado tres genes ras: el H-ras, $\mathrm{K}$-ras y $\mathrm{N}$-ras, los cuales presentan distintas mutaciones, afectando principalmente la actividad GTPasa de las proteínas ras. La mayoría de ellas afectan al codón I2. Múltiples tumores presentan mutaciones ras y la frecuencia varía ampliamente en cada uno de ellos. Por ejemplo, los carcinomas son K-ras mientras que los tumores hematopoyéticos son $\mathrm{N}$-ras y en otros tumores son muy raras o inexistentes. En tumores odontogénicos 
como el fibroma ameloblástico, mixomas odontogénicos y ameloblastoma se ha visto una sobreexpresión del gen ras en el componente epitelial, en comparación con el epitelio normal del diente en desarrollo. La mutación del K-ras en el codón 12 ha sido detectada en solo uno de 23 ameloblastomas $^{26}$ Por lo cual, a pesar de que las mutaciones ras son extremadamente frecuentes en la mayoría de los tumores, su presencia no es primordial en la carcinogénesis de los tumores odontogénicos ${ }^{27}$.

Los genes Myc y Fos pertenecen a un grupo de genes implicados en la trascripción de proteínas nucleares, los cuales se activan rápidamente cuando las células en reposo reciben la señal que promueve su división. Dentro de los productos de los oncogenes myc, el c-myc se encuentra en prácticamente todas las células eucariotas y pertenece a los genes de respuesta de crecimiento precoz e inmediato, no solo controla el crecimiento celular si no que también puede dirigir la muerte celular través de la apoptosis. La sobreexpresión de la proteína myc ocasiona una transcripción continua de genes diana que puede generar, como se expresó anteriormente, su transformación neoplásica. Un trabajo de de Dodds y $\mathrm{col}^{28}$ revela una alta expresión de los genes myc y K-ras en tumores odontogénicos y por otra parte un trabajo de Heikinheimo y $\mathrm{col}^{29}$ reportan una sobreexpresión del oncogén Fos en ameloblastomas. Estos hallazgos sugieren que ambos oncogenes juegan un papel en la patogenia de algunos tumores odontogénicos a través de una desregulación de la proliferación celular.

La telomerasa es una enzima que estabiliza la longitud del telómero al añadirse a los extremos de los cromosomas. Durante el envejecimiento celular se encuentran acortados y por el contrario en las células neoplásicas inmortales se reactiva la acción de la telomerasa y no se acortan los telómeros participando en la formación del tumor. La activación de la telomerasa se ha demostrado en más tumores humanos malignos. Repeticiones teloméricas en protocolos de ensayo (TRAP) demostraron positividad en los ameloblastomas, lo cual sugiere que la activación de la telomerasa se asocia con la tumorogénesis del epitelio odontogénico. La reactividad inmohistoquímica de la telomerasa en ameloblastomas muestra un patrón similar a la de la proteína c-myc, un oncogen que activa directamente la transcripción de la telomerasa, sugiriendo que esta oncoproteína induce la actividad de la telomerasa en los ameloblastomas ${ }^{30}$.

Entre las muchas técnicas de laboratorio utilizadas para los diagnósticos e investigaciones de quistes y tumores odontogénicos, la inmunohistoquímica es un método de estudio específico de gran valor en la Anatomía Patológica. Se basa en la utilización de anticuerpos, para poder demostrar proteínas reguladoras del crecimiento celular y una gran variedad de antígenos presentes en las células y tejidos. El espectro de anticuerpos disponibles comercialmente crece día a día y actualmente es posible encontrar marcadores para una amplia gama de antígenos, lo que facilita el estudio de las distintas patologías.

La importancia del uso de las mismas, radica en que pueden señalar las moléculas o productos proteicos implicados en la tumorogénesis.

Los marcadores inmunohistoquímicos pueden detectar estas alteraciones que se expresan durante distintas fases del ciclo celular y permiten indicar la proliferación activa de las células o la posibilidad de su transformación oncogénica, como la expresión del antígeno de proliferación nuclear (PCNA), la expresión proteica de proliferación celular (Ki-67) y el gen supresor de tumor (p-53) y se utilizan como indicadores de valor pronóstico en el desarrollo de quistes y tumores odontógenos.

Los quistes odontogénos descriptos y reclasificados por la OMSI7 destacan su origen en diferentes etapas de la odontogénesis y puntualmente, en la clasificación histogenética descripta por Sapp $^{31}$ a partir del reconocimiento embriológico, identifica sus orígenes de acuerdo a los restos epiteliales del cual se originen. Así pues, se ha observado que de la cubierta epitelial intracavitaria de los quistes dentígeros y queratoquistes a veces se pueden observar variaciones en la histoarquitectura epitelial, tales como hiperplasia epitelial, elongación de la redes de cresta y/o alteraciones de la unión epitelio-conectivo y entonces, la utilización de inmunotinciones contribuyen significativamente como colaboradores para identificar su comportamiento y probable transformación neoplásica. Algunos trabajos de Bascones y Llanes ${ }^{32}$ en quistes odontógenos señalan que en ausencia de componente inflamatorio, el revestimiento epitelial se encuentra conservado y muestran positividad para las citoque- 
ratinas (CK), el antígeno epitelial de membrana (EMA) y el antígeno de proliferación nuclear (PCNA) alcanzan valores muy bajos menores al $5 \%$ y carecen de significancia pronóstica. Pero cuando existe proliferación celular, pueden alcanzar valores superiores al $10 \%$ de PCNAy se tornan significativos que, en conjunto con las alteraciones morfológicas descriptas por Vickers y Gorlin, hace más de dos décadas, como: la hipercromasia nuclear, la vacuolización citoplasmática, la polarización nuclear inversa, la pérdida de cohesión ylo la fibroplasia laminar, los convierten a éstos marcadores en buenos predictores del aumento de la actividad mitótica y probable trasformación ameloblástica. Cuando se produce una transformación ameloblástica en un quiste odontógeno, el inmunofenotipo muestra negatividad para EMA y positividad para PCNA a diferencia del epitelio quístico no transformado, por lo que se puede interpretar que ambos antígenos son útiles para la detección de los mismos. También son recomendables otros marcadores de proliferación celular, como el (KI-67) y el antígeno carcinoembrionario (ACE) ya que son buenos predictores pronósticos y su estudio en el epitelio de los quistes maxilares y ameloblastomas son útiles para evaluar la potencialidad agresiva y en los quistes, detectar cambios incipientes de transformación ameloblastomatosa y su probabilidad de malignización.

El crecimiento celular comienza por la unión de un factor de crecimiento con un receptor específico que puede encontrarse en la superficie celular, en el citoplasma o en el núcleo. Durante el ciclo celular, las células quiescentes primero deben hacer una transición desde la fase $\mathrm{GO}$ a GI, que funciona como una puerta de entrada al ciclo celular. Esta transición implica la activación de transcripción de un grupo amplio de genes, incluyendo diversos protooncogenes $y$ genes requeridos para la síntesis de ribosomas y la trasducción de proteínas y entonces las células pueden entrar en GI desde G0 o tras completar la mitosis (en el caso de células en replicación continua). Las células que iniciaron el ciclo celular avanzan alcanzando un estado crítico en la transición GI/S conocido como punto de restricción, el cual una vez superado, las células normales entran de forma irreversible a la replicación de su ADN. En este punto de restricción la actividad se encuentra regulada por proteínas como las ciclinas $y$ enzimas relacionadas denominadas cinasas dependientes de las ciclinas (CDK) y de sus inhibidores que tienen un papel importante en el control del ADN para evitar su progresión en el ciclo celular en caso de que exista algún tipo de daño, activando los mecanismos apoptóticos. En la fase G2, la célula se prepara para la división llegando a un segundo punto de control conocido como G2/M en el cual se vigila que la replicación el ADN y se controla si la célula puede iniciar la mitosis, garantizando que el ciclo celular se complete en forma correcta.

La ciclina DI, junto con las cinasa dependientes de ciclinas (CDK), están encargadas de controlar el tránsito en el ciclo celular desde la fase GI a $S$ en el llamado punto de restricción. La desregulación y la sobreexpresión de la ciclina DI se ha puesto de manifiesto en muchos tumores de diversa histogénesis y se lo ha relacionado con el rápido crecimiento y el aumento de la actividad proliferativa, como así también con la agresividad histológica y la capacidad metastásica33. Un estudio con inmunohistoquimica realizado por Kumar y col. ${ }^{34}$ sobre 39 casos de ameloblastomas y 7 casos de tumor odontogéno adenomatoide demostraron una tinción positiva para la expresión de ciclina DI en el $79,5 \%$ de Ameloblastomas y solo un $63,3 \%$ en tumores Odontógenos Adenomatoides. La marcación evidenció una coloración marrón en el sitio del antígeno diana, localizado en el núcleo y en el citoplasma expresándose de manera significativa en células basales periféricas y en células centrales del retículo estrellado de los Ameloblastomas con variable intensidad, 3 mostraron una tinción intensa, 12 una tinción moderada y 16 casos con una tinción leve. Por otro lado, en los Tumores Odontogénicos Adenomatoides solo se evidenció tinción nuclear. Así, la inmunotinción con ciclina DI demostró su participación en la proliferación de estos tumores y sus patrones de expresión fueron independientes del comportamiento biológico. Crivellini y col..$^{35}$ realizaron un estudio con PCNA en Tumores Odontogénicos Adenomatoides, observando inmunoreactivdad en las áreas más sólidas, con patrón tubular y rosetoide, observando similitudes con los hallazgos de Kumar con la ciclina DI, señalando mayor actividad en esas áreas.

El patrón de tinción heterogénea observado en los casos positivos varió de leve 
a intenso en los mismos campos microscópicos, posiblemente debido a las variaciones no solo en los niveles de proliferación celular, si no también en los niveles de proteínas durante la progresión del ciclo celular. Por ello se consideró la intensidad de la tinción en los mismos.

Por lo tanto, se considera que las Ciclinas y las CDK tienen un importante papel en el control del ciclo celular, su expresión de variable intensidad en el ameloblastoma, también se observa en el núcleo en las células parabasales del queratoquiste odontogénico y en los quistes dentígeros y los quistes radiculares, lo cual apoya que la mayor expresión de la ciclina DI se asocia con las áreas de mayor actividad proliferativa de estas lesiones odontogénicas ${ }^{36}$ y cualquier alteración en las mismas puede conducir a la oncogénesis.

Otro gen supresor bien estudiado es el p-53 y está situado en el cromosoma 17p|3.I. Esta proteína se encuentra en el núcleo controlando la transcripción de otros genes y ocasionan la detención del ciclo celular al final de la fase GI, permitiendo la reparación del ADN provocado por el mutágeno y/o en caso contrario induce la activación de la apoptosis. La pérdida homocigótica del gen p53 aparece en casi todos los tipos de cáncer y en un trabajo de Kumamotol 6 de la División Patología Oral del Departamento de Medicina Oral y Quirúrgica de la Escuela de Graduados de Dentistas de la Universidad de Tohoko, Sendai - Japón, reveló una intensa reactividad con inmunotinciones para el p53 en casos de ameloblastomas, ameloblastomas malignos, carcinoma intraóseo primario y fibrosarcoma ameloblástico. La regulación de p-53, con MDM2 y p-I4 ARF fueron expresados en ameloblastomas, tumor odontogénico adenomatoide, ameloblastomas malignos y el carcinoma odontógeno de células claras y sobre expresadas con MDM2 y p-I4 ARF en ameloblastomas lo cual sugiere un factor de asociación morfo-estructural $y$ cito-diferenciación.

Existen muchos virus ADN y ARN oncogénicos en una amplia variedad de animales y cada vez aparecen más evidencias que algunos tumores humanos son producidos por virus, identificables con técnicas de inmomarcación como el Virus de Ebstein Bar (EBV) implicado en el Linfoma de Burkitt y el Virus del Papiloma Humano (HPV) 37,38,39 que existen mas de 75 tipos de cepas bien identificadas y son capaces de produ- cir verrugas y carcinomas de células escamosas por su epitelio-trofismo. Sin embargo, a pesar de algunas publicaciones sobre su presencia en ameloblastomas, el papel en la etiopatogenia de los tumores odontógenicos, sigue siendo controvertido ${ }^{40}$.

Las técnicas inmunohistoquímicas también sirven para señalar los genes reparadores del ADN. Estos no son oncogénicos por si mismos, pero permiten que se produzcan mutaciones en otros genes durante el proceso de división celular normal. Un trabajo de Castrilli y colaboradores utilizaron el hMSH2 y el hMLHI que fueron detectados en ameloblastomas y se visualiza la inmunomarcación en la parte periférica de las células neoplásicas, sugiriendo participar en el desarrollo y progresión del tumor odontogénico ${ }^{41}$.

Considerando otros factores como los angiogénicos, la evaluación inmunohistoquímica con CD34 de la microvasculatura y de las células endoteliales, muestran una marcada vascularización en los gérmenes dentarios y también en los ameloblastomas. Simultáneamente, el incremento de la expresión del factor de crecimiento del endotelio vascular (VEGF), sugiere que constituye un importante mediador de la angiogénesis tumoral en los ameloblastomas y podrían estar asociados a la transformación maligna del epitelio odontogénico ${ }^{42}$.

Finalmente en un artículo de de Zhang y colaboradores ${ }^{43}$, investigaron en 12 quistes, la expresión de la $\mathrm{SHH}$ en los Quistes Odontogénicos Glandulares (GOCs) y Quistes Dentígeros (QD), también utilizaron el $\mathrm{PCH}$, el SMO y el factor de transcripción GLII. Tanto en el GOCs como en los QD fueron detectados en la superficie epitelial, demostrando que cada uno de los genes de las señalizaciones se expresa con valores similares en el revestimiento de los quistes. La expresión de SHH, PTT, SMO y GLII fue significativamente mayor en los epitelios que en los fibroblastos subepiteliales $(\mathrm{P}<0,0 \mathrm{I})$ aunque no estableció una estadística significativa entre los diferentes tipos de quistes observados.

Estos resultados sugieren que las proteínas de la vía de señalización de $\mathrm{SHH}$ se localizan predominantemente dentro de los componentes epiteliales de GOCs. La vía de señalización de la SHH puede jugar un papel en la formación del revestimiento epitelial. 


\section{Conclusión}

Las técnicas inmunohistoquímicas, actualmente establecidas dentro de los exámenes de rutina y ampliamente utilizadas, constituyen un importante medio de la Anatomía Patológica que ayuda focalizar el sitio de la lesión identificando productos celulares y marcadores de superficie $y$ en conjunto con los hallazgos morfológicos y las variables clínico-radiográficas favorecen una mejor comprensión de la naturaleza y comportamiento biológico de estas lesiones.

Se concluye enfatizando que se debe conocer las alteraciones moleculares subyacentes para predecir la evolución de estas patologías que redunda en beneficio de una terapéutica adecuada.

\section{Bibliografía}

I. Hanahan, D.; Weinberg, R. A. "Hallmarks of cancer: The Next generation" Cell, March 4, 201 I 20II Elsevier Inc. I44, 646 - 670.

2. Hanahan, D.; Weinberg, R.A. The hallmarks of cancer. Cell, 2000, 100, 57 - 70.

3. Aguirre-Ghiso, J. A. Models, mechanisms and clinical evidence for cancer dormancy. Nat. Rev. 2007. Cancer 7, 834 - 846.

4. Ahmed, Z., and Bicknell, R. Angiogenic signalling pathways. Methods. Mol. Biol. 2009. 467, 3 - 24.

5. Folkman, J. Role of angiogenesis in tumor growth and metastasis. Semin. Oncol.2002; 29 (6, Suppl 16), 15 - 18.

6. Folkman, J. Angiogenesis. Annu. Rev. Med. 2006: 57, I - 18.

7. DeNardo, D.G., Andreu, P., and Coussens, LM. Interactions between lymphocytes and myeloid cells regulate pro- versus anti-tumor immunity. Cancer Metastasis Rev.2010: 29, 309 - 316.

8. Collado, M., and Serrano, M. Senescence in tumours: evidence from mice and humans. Nat. Rev. Cancer 2010. 10, 51 - 57.

9. Colotta, F., Allavena, P., Sica, A., Garlanda, C., and Mantovani, A. Cancer-related inflammation, the seventh hallmark of cancer: links to genetic instability. Carcinogenesis 2009.30, 1073 - $108 \mathrm{I}$.

10. Klymkowsky, M.W., and Savagner, P. Epithelialmesenchymal transition: a cancer researcher's conceptual friend and foe. Am. J. Pathol.2009. 174 , 1588 - 1593

II. Fang, S., and Salven, P. Stem cells in tumor angiogenesis. J. Mol. Cell.Cardiol.20I I. 50, 290-295.

12. Kalluri, R., and Zeisberg, M. Fibroblasts in cancer. Nat. Rev. Cancer; 2006. 6, 392 - 40I.
13. Jones, P. A., and Baylin, S. B. The epigenomics of cancer. Cell,2007: 128, 683 - 692.

14. Jones, R. G., and Thompson, C. B. Tumor suppressors and cell metabolism: a recipe for cancer growth. Genes Dev.2009: 23, 537 - 548.

I5. Joyce, J. A., and Pollard,J. W. Microenvironmental regulation of metastasis. Nat. Rev. Cancer 2009: 9, 239 - 252.

16. Kumamoto, H. "Molecular pathology of odontogenic tumors". J.Oral Pathol Med 2006.35: 65 $-74$.

17. Barnes L; Evenson J.; Reichart P.; Sidranski D."World Health Organization Classification of Tumors Pathology and Genetics of Head and Neck Tumoours" IARC Press. Lyon, 2005.

18. Li, T. J. "The Odontogenic Keratocyst: a cyst or a Cystic Neoplasm?". J Dent Res ,201 I, 90 (2): 133 - 142.

19. Gu XM, Zhao HS, Sun LS, Li TJ. “ PATCH mutations in sporadic and Gorlin-Syndrome-related odontogenic keratocyst" J Dent Res 2006. 85: 859 - 863.

20. Gomes C. C., Diniz M. G., Gomez R. S.: "Review of the Molecular Pathogenesis of the Odontogenic Keratocyst" Oral Oncol , 2009a.45: 10II - 1014.

21. Gomes C.C., Oliveira Cda S., Castro W.H., de Lacerda JC., Gomez RS. "Clonal Nature of Odontogenic Tumors"] Oral Pathol Med, 2009 b. $38: 397$ - 400 .

22. Mendes R. A., Carvalho J. F., van der Waal, Y. "Biological pathways involved in the agressive behavior of the keratocystic odontogenic tumor and possible implications for molecular oriented treatment-an overview" Oral Oncol. 2010. 46: 19 - 24. 
23. Fan Z., Li J., Du J., Zhang H., Shen Y., Wang C. Y., et al. "A missense mutation in PTCH2 underlies dominantly inherited NBCCS in a Chinese family". J Med Genet 2008, 45: 303 - 308.

24. Pastorino L, Ghiorzo P, Nasti S, Battistuzzi L, Cusano R, Marzocchi C, et al. "Identification of a SUFU germline mutation in a family with Gorlin syndrome"J. Med Genet Part A. 2009. I49: 1539 - 1543.

25. Henley J, Summerlin DJ, Tomich C, Zhang S, Cheng $L$. "Molecular evidence supporting the neoplastic nature of odontogenic keratocyst: a laser capture microdissection study of 15 cases". Histopathology ,2005. 47: 582 - 586.

26. Kumamoto H, Takahashi N, Ooya K. K-Ras gene status and expression of Ras/mitogen-activated protein kinase (MAPK) signaling molecules in ameloblastomas. J Oral Pathol Med 2004; 33: 360 $-7$.

27. Kumar,V; Abbas,A; Fausto,N; Aster,J. Robbins y Cotran Patología estructural y funcional. Octava edición. Barcelona, España. Editorial Elsiever. 2010,(Cap.3) 80 - 89 y (Cap 7) 276 - 318.

28. Dodds AP, Cannon RE, Suggs CA, Wright JT. mRNA expression and phenotype of odontogenic tumours in the v-Ha-ras transgenic mouse. Arch Oral Biol 2003; 48: 843 - 50.

29. Heikinheimo K, Jee KJ, Niini T, et al. Gene expression profiling of ameloblastoma and human tooth germ by means of a cDNA microarray. J Dent Res 2002; 8I: 525 - 30.

30. Kumamoto H, Kinouchi Y, Ooya K. Telomerase activity and telomerase reverse transciptase (TERT) expression in ameloblastomas. J Oral Pathol Med 200I; 30: 23 I - 6.

3I. Sapp J; Eversole L; Wysocki, G. "Patología Oral y Maxilofacial Contemporánea". Segunda Edición. Editorial Elsevier-Mosby. 2004; 2, 45 - 61.

32. Bascones,J y Llanes,F. "Factores pronósticos en quistes radiculars y foliculares" Hospital Clínico de San Carlos. Madrid. Comunicación III Congreso Virtual Hispanoamericano de Anatomía Patológica" feb. 2000.

33. Todd R, Hinds PW, Munger K, Rustgi AK, Opiz OG, Suliman Y, et al. "Cell cycle dysregulation in oral cancer". Crit Rev Oral Biol. Med 2002;|3: $51-61$.
34. Kumar $H$, Vandana $R$, Immunohistochemical expression of cyclin DI in ameloblastomas and adenomatoid odontogenic tumors.Journal of Oral and Maxillofacial Pathology: Sep - Dec 201 I. Vol. 15 Issue 3.

35. Crivelini MM, Soubhia AM, Felipini RC. Study on the origin and nature of adenomatoid odontogenic tumor by immunohistochemistry. J Appl Oral Sci 2005; 13: 406 - 12.

36. De Vicente JC, Torre-lturraspe A, Gutiérrez AM, Lequerica-Fernández P. Immunohistochemical comparative study of the odontogenic keratocysts and other odontogenic lesions. Med Oral Patol Oral Cir Bucal 20 10; 15: e709 - 15.

37. Sand L., Jalouli J., Larsson P. A., Magnusson B., Hirsch JM. Presence of human papilloma viruses in intraosseous ameloblastoma. J Oral Maxillofac Surg 2000; 58: II 29 - 34.Jang HS, Cho JO, Yoon CY, Kim HJ, Park JC. Demonstration of EpsteinBarr virus in odontogenic and nonodontogenic tumors by the polymerase chain reaction (PCR). J Oral Pathol Med 200I; 30: 603 - I0.

38. Namin AK, Azad TM, Eslami B, Sarkarat F, Shahrokhi M, Kashanian F. A study of the relationship between ameloblastoma and human papilloma virus. J Oral Maxillofac Surg 2003; $61: 467$ $-70$.

39. Migaldi M, Pecorari M, Rossi G, et al. Does HPV play a role in the etiopathogenesis of ameloblastoma? An immunohistochemical, in situ hybridization and polymerase chain reaction study of 18 cases using laser capture microdissection. Mod Pathol 2005; 18: 283 - 9.

40. Castrilli G, Piantelli M, Artese L, et al "Expression of hMSH2 and hMLHI proteins of the human DNA mismatch repair system in ameloblastoma". J.Oral Pathol Med 200I; 30: 305 - 8.

4I. Kumamoto,H.Ogki K, Ooya. "Association between vascular endothelial growth factor (VEGF) expression and tumor angiogenesis in ameloblastoma". J.Oral Pathol Med 2002. 3I: 28 $-34$.

42. Zhang, I., Sun, Z. L,Chen, X. M., Chen; Z; "Immnohistochemical expresión of SHH, PTC; SMO and GLII in glandular odontogenic cysts and dentigerous cysts" Oral Diseases, $2010-16$, $818-822$. 